\title{
On-Chip Capillary Electrophoresis (Chip-CE) with Optical On-Chip Leaky-Waveguide-Based Detection
}

\author{
Christoph Doering, Matthias Grewe, Ingo Steingoetter, Henning Fouckhardt \\ Integrated Optoelectronics and Microoptics Research Group, Physics Department, Kaiserslautern University of \\ Technology, Kaiserslautern, Germany \\ Email: cdoering@physik.uni-kl.de
}

Received 20 January 2014; revised 25 February 2014; accepted 5 March 2014

Copyright @ 2014 by authors and Scientific Research Publishing Inc.

This work is licensed under the Creative Commons Attribution International License (CC BY). http://creativecommons.org/licenses/by/4.0/

(c) (7) Open Access

\begin{abstract}
Capillary electrophoresis (CE) suffers from a relatively small sensitivity-at least in case of optical detection transversely to the capillary axis due to the small capillary inner diameters in the range of $50-100 \mu \mathrm{m}$. Different concepts like bubble, U-, or Z-cells have been used to tackle that problem already in the nineties of the last century. But the $U$ - and Z-cells have typically been extra cells with larger inner channel diameters and no optimization for optical waveguiding and the bubble cell per se did not allow for optical waveguiding. In the case of on-chip capillary electrophoresis (chip-CE) a U-cell can be implemented quite easily on the chip. Here we show how leaky optical waveguiding can be employed to improve optical detection. Proper U-channel design and preparation by wet-chemical etching of the fused silica sub- and superstrate, making the $U$-channel bend a part of the optical input lens system, can help to achieve high coupling efficiency with loss coefficients around $2 \mathrm{~dB}$ and low waveguiding loss.
\end{abstract}

\section{Keywords}

On-Chip Capillary Electrophoresis; Optical Aetection; Leaky Waveguides

\section{Introduction}

Sensitive optical detection is always a problem for capillary electrophoresis due to the small inner diameters of the capillary fluid channel. In case of an optical path transverse to the capillary axis, a bubble cell was used to increase sensitivity [1]-[3], i.e. a local increase of the capillary diameter by a factor of $3-5$. One problem with the bubble is that different light rays traverse the bubble via very different paths, some with no interaction with 
the fluid at all, others with an optical interaction length equal to the full inner bubble diameter, to mention only both extremes. Another concept employs $\mathrm{U}$ - or Z-cells, where the middle part of the $\mathrm{U}$ or of the $\mathrm{Z}$ is used as an optical path [4]-[7]. But typically U- or Z-cells have larger inner channel diameters than the capillary itself and are extra parts of the separation column, which have to be physically attached to the capillary somehow. This leads to dead volumes and thus to a reduction in chromatographic resolution.

The use of on-chip technologies does not prevent these problems, because still the light path has to be optimized. Much earlier optical waveguiding had been proposed to increase interaction path lengths in cases of integrated solutions [8] [9]; but the interaction had been performed via the evanescent parts of the optical field only. i.e. the light had mainly been guided in the capillary wall, while the field portion used for interaction with the analyte showed an exponential decay into the fluid (the so-called evanescent field), giving weaker interaction than desired. This will be unavoidable, as long as waveguiding is only understood as the usual waveguiding by total internal reflection, where the main portion of the light field is propagating within an area of high refractive index-like 1.47 for fused silica. In this case the often aqueous analyte solution with a refractive index around 1.33 can only serve as a waveguide cladding with an evanescent field.

Therefore, leaky waveguiding is an option [10]-[16], where the waveguide core is free to have any refractive index and transverse layers or regions act as coupled transverse optical resonators for grazing light incidence, redirecting the field into the core, as depicted in Figure 1. The notion "leaky waveguide" stems from that part of the light power not redirected, causing unavoidable loss. For acceptably low loss waveguiding distances are limited to centi- or even millimeters. But for inner capillary diameters of 50 - $100 \mu \mathrm{m}$ the transverse resonators and their production expenditures are not even necessary [17]; the Fresnel reflections at the single interface between the fluid and the fused silica wall suffice to achieve acceptably low waveguiding loss.

In [17] we also showed exemplarily for hyper Rayleigh scattering (HRS [18] [19])—and a planar chip with fused silica substrate and superstrate- that this concept can enhance detection efficiency by at least a factor of 20. This is due to the long path of high pump intensity along the channel waveguide then with a length of $15 \mathrm{~mm}$ and due to the low reabsorption of the HRS light on its short way transversely out of the then $100 \mu \mathrm{m}$ broad channel- Here we extend this leaky waveguide approach by showing that the round cross-section wet-chemically etched into the fused silica substrate/superstrate can work as a dispersing lens at the waveguide bend. This lens and an external lens system are jointly optimized to improve optical input efficiency.

\section{Chip}

\subsection{Design}

The complete planar CE-Chip is shown in Figure 2 with a size comparison to a Euro cent coin (16 mm coin diameter). The optical path is marked by the arrows in Figure 2 .

\subsection{Technology}

Figure 3 gives an overview of the technological process to realize the chip by wet-etching both the substrate and the superstrate in the same way with mirror-inverted channel patterns. First both have to be thoroughly cleaned and prepared for photoresist wetting. Then both are spin-coated with the photoresist; different resist options are possible [17] [20] [21]. Then the latter is exposed to ultraviolet light in the pattern of the channel lay-out, developed, and hardbaked. The opening in the photoresist layer allows for wet-chemical etching of the substrate and superstrate by buffered hydrofluoric acid. The wet-etching causes half-round cross-sections (giving half-cylindrical channels). After photoresist descum in an oxygen plasma the fluid inlet holes have to be drilled or sand-blasted. Then sub- and superstrate are aligned and bonded by pressure and heat treatment with severe alignment tolerances of less than $1 \mu \mathrm{m}$ [22]. At the bottom of Figure 3 a microscope image of a channel crosssection after bonding of sub- and superstrate is shown; as intended no optical interface between both chip halves can be observed. (Note that in this case the chip has only been transversely sawed to make the microscope image of Figure 3 possible.)

\section{Longitudinal Optical Coupling-Channel Bend as Part of Input Lens System}

Figure 4 is giving a combination of two microscope images and a sketch (not to scale), illustrating that the waveguide bend is part of the input-coupling lens system together with an external achromatic lens set-up-like in 


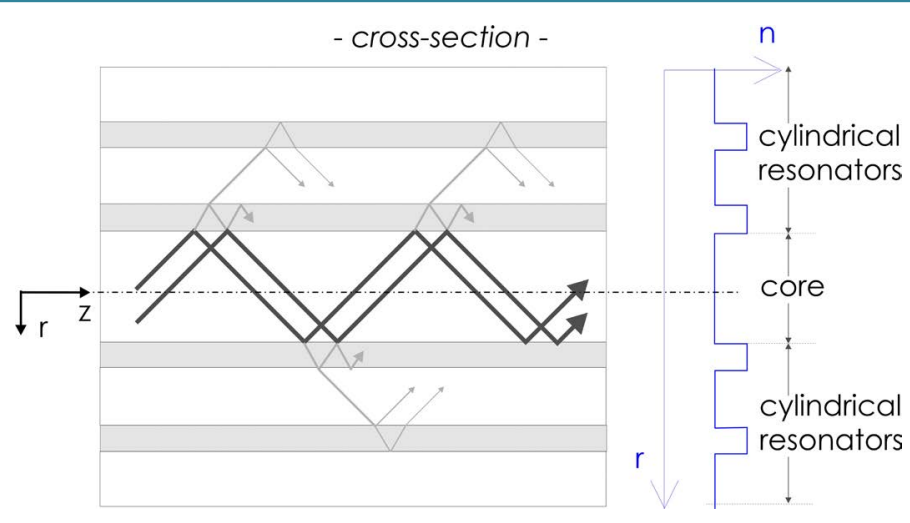

Figure 1. Illustration of leaky optical waveguiding; $r$ is the radial coordinate, $n$ denotes the refractive index.

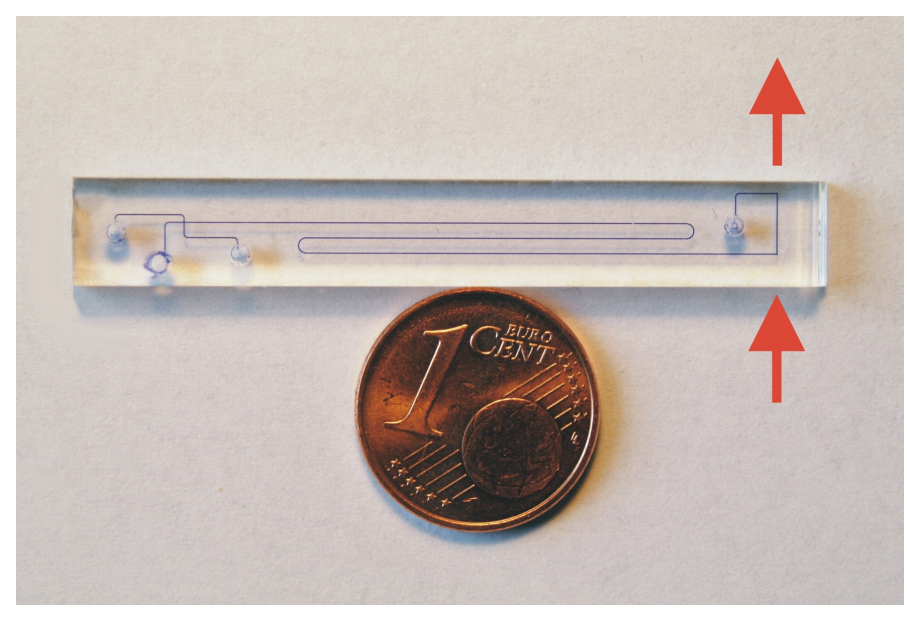

Figure 2. CE chip with leaky waveguide detection path.

the case of a Galilei-type telescope. (Note, that these microscope images show a slightly other chip version with an oblique feeding capillary as opposed to the case in Figure 2.) For the micrograph on the right in Figure 4 the capillary has been completely filled by the dye/chromophore Rhodamine 6G and illuminated/excited from outside with the light of an $\mathrm{Ar}^{+}$laser. The fluorescence extends over the whole straight channel, because the exciting beam is guided in this channel.

For a channel diameter of $\approx 65 \mu \mathrm{m}$ and the optimized optical system, the results averaged over typical solvents (like water, methanol, and dioxane) and over the wavelengths of the visible spectrum, the waveguide insertion loss is about $2.0 \mathrm{~dB}$ for optical input (and output) coupling and about $0.07 \mathrm{~dB} / \mathrm{mm}$ due to the leaky character of the optical waveguiding, i.e. f.e. $0.70 \mathrm{~dB}$ for a $10 \mathrm{~mm}$ long waveguide section, giving a loss of about $2.70 \mathrm{~dB}$ altogether [17] [22].

\section{CE Fluorescence Detection}

To show the potential of this approach exemplarily a capillary electrophoresis is run with a solution of TRIS(hydroxymethyl) aminomethane as the solvent and the chromophores Rhodamine B (R B) and Fluorescein 27 (F 27) as solutes with similar molecular backbones. The separation column is $92 \mathrm{~mm}$ long; the concentration of the solution is $14.2 \mu \mathrm{mol}$. A voltage of $3.25 \mathrm{kV}$ is applied along the separation channel, giving en electric field strength of $35 \mathrm{~V} / \mathrm{mm}$ - The excitation of the dyes is again realized with the beam of an $\mathrm{Ar}^{+}$laser coupled into and waveguided in the optical channel, similar to the situation in Figure 4, but now with the dyes as short plugs within the solvent electrophoretically formed and moving along the channel. Fluorescence detection is performed with the help of a glass fiber tip butt-coupled perpendicularly to the chip after an optical channel length of $5.0 \mathrm{~mm}$ and connected to a fiber-pig-tailed grating spectrometer with a spectral resolution of $1.2 \mathrm{~nm}$. 


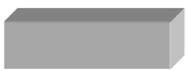

1. cleaning - Chromosulphoric-acid @ $150^{\circ} \mathrm{C}(>5 \mathrm{~h})$
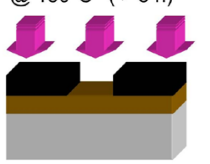

7. exposure - mask spacing 5-7 $\mu \mathrm{m}$ for microchannel

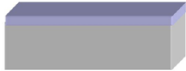

2. rinsing

- deionized $\mathrm{H}_{2} \mathrm{O}+$ ultrasound

- dried w. filtered $\mathrm{N}_{2}$

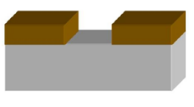

8. development - in diluted AZ400K

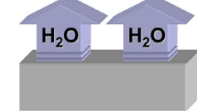

3. annealing - $300^{\circ} \mathrm{C}(>8 \mathrm{~h})$ - cool to $90^{\circ} \mathrm{C}$
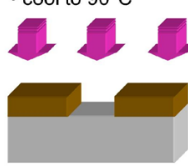

9. flood exposure - produces indene - produces inden
carboxylic acid

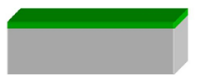

4. Hexamethyldisilazan - $\mathrm{H}_{2} \mathrm{O}$ removed - improved PR adhesion

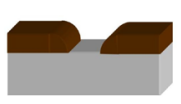

10. hardbake - crosslinking the novolak resin w. the carboxylic acid (@) $120-180^{\circ} \mathrm{C}$ )

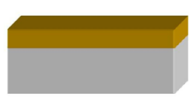

5. photoresist

- 1,4 um AZ ${ }^{\circledR 5214}$

(@ 4000 U/min)

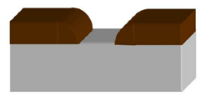

11. pre-etch bake

- 1 hour before etching

procedure

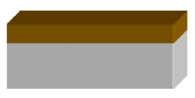

6. prebake - evaporation of solvent

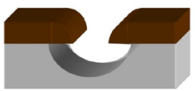

12. etching - buffered HF-solution

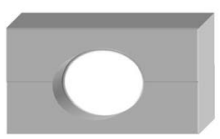

13a. bonding - annealing after aligning and contacting sub- and superstrate

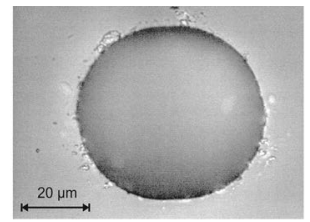

13b. Bonding photograph of a nearly circular channel cross section

Figure 3. Sketch of technological process for CE-chip realization. Other photoresist (PR) options are possible.

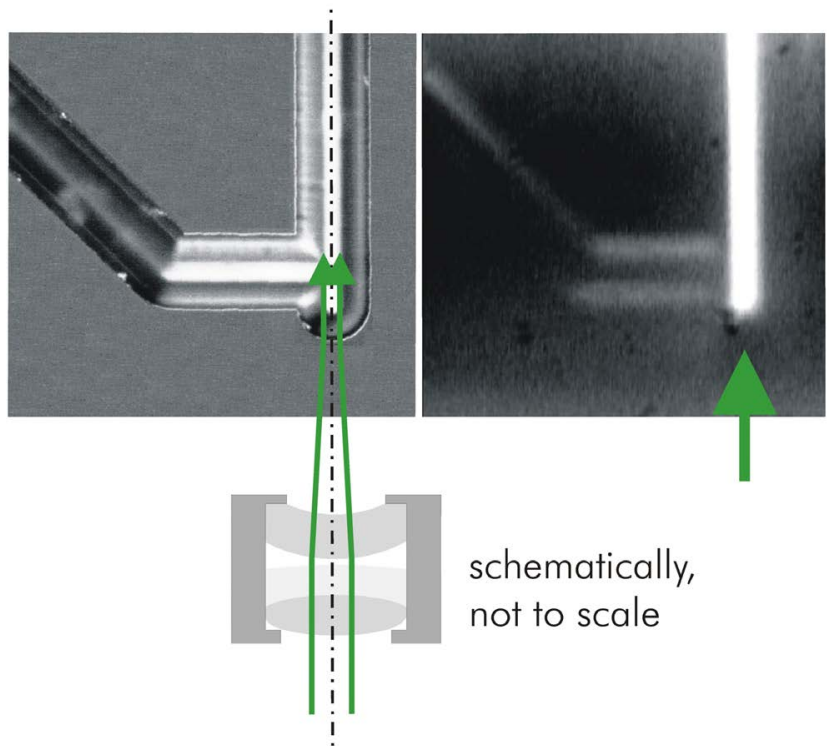

Figure 4. Channel bend with optical function as a dispersing lens forming a lens system with the external achromatic lens set-up, i.e. a Galilei-type telescope. On the right fluorescence of a Rhodamine 6G dye solution-here fully filling the channelexcited by $\mathrm{Ar}^{+}$laser light can be observed. The inner channel diameter is about $65 \mu \mathrm{m}$ here.

The CE result is shown in Figure 5. Two fluorescence peaks demonstrate a temporal and spatial separation of the two markers Rhodamine and Fluorescein. The Rhodamine plug reaches the position of the fiber tip for detection first-after $85 \mathrm{~s}$. The maximum of its fluorescence signal is at a wavelength of $566 \mathrm{~nm}$. About $60 \mathrm{~s}$ later the Fluorescein plug reaches the detection fiber. Here the fluorescence maximum is at $547 \mathrm{~nm}$.

\section{Conclusions}

It is shown that leaky waveguiding can be used advantageously in on-chip capillary electrophoresis (chip-CE) 


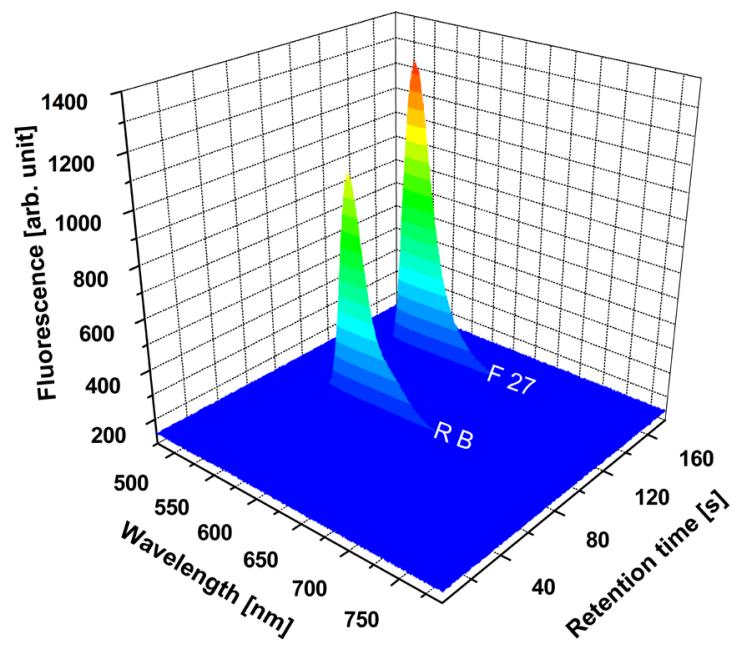

Figure 5. CE separation result for the chromophores Rhodamine B (R B) and Fluorescein 27 (F 27). The fluorescence maximum for $\mathrm{R} B$ is at a wavelength of $566 \mathrm{~nm}$, while that for $\mathrm{F} 27$ is at $547 \mathrm{~nm}$. The retention time for R B is $85 \mathrm{~s}$, while that for $\mathrm{F} 27$ is $145 \mathrm{~s}$.

—exemplarily for the excitation light in fluorescence detection. Efficient input coupling can be achieved by incorporation of a wet-chemically etched waveguide bend. It acts as a dispersing lens and forms a Galilei-type telescope together with an external achromatic lens set-up.

In principle, the waveguiding concept can also be employed for other types of separation techniques and other methods of optical detection, like absorption spectroscopy. In the latter case f.e. the waveguided light can be coupled out of the channel again in the longitudinal direction with another Galilei-type micro telescope.

\section{Acknowledgements}

The work was financially supported by the German Research Foundation (DFG, Deutsche Forschungsgemeinschaft) under contract FO157/15.

\section{References}

[1] Hewlett-Packard (Now Agilent Technologies) (1992) Product Specifications on Capillaries with Bubble Cell.

[2] Kaltenbach, P. (1995) A High-Sensitivity Diode Array Detector for On-Column Detection in Capillary Electrophoresis. HP Journal, 46, 20-24.

[3] Agilent Technologies (1997) Agilent Technologies Technical Note. 5965-5984.

[4] Bruno, A.E., Gassmann, E., Pericles, N. and Anton, K. (1989) On-Column Capillary Flow Cell Utilizing Optical Waveguide for Chromatographic Applications. Analytical Chemistry, 61, 876-883.

[5] Verpoorte, E., Manz, A., Luedi, H., Widmer, H.M., van der Schoot, B.H. and de Rooij, N.F. (1991) A Novel Optical Detector Cell for Use in Miniaturized Total Chemical Analysis Systems. Proceedings of the International Conference on Solid-State Sensors and Actuators, San Francisco, 24-27 June 1991, 796-799.

[6] Verpoorte, E., Manz, A., Luedi, H., Bruno, A.E., Maystre, F., Krattiger, B., Widmer, H.M., van der Schoot, B.H. and de Rooij, N.F. (1992) A Silicon Flow Cell for Optical Detection in Miniaturized Total Chemical Analysis Systems. Sensors and Actuators B Chemical, 6, 66-70. http://dx.doi.org/10.1016/0925-4005(92)80032-S

[7] Moring, S.E., Reel, R.T. and van Soest, R.E.J. (1992) Optical Improvements of a Z Shaped Cell for High-Sensitivity UV Absorbance Detection in Capillary Electrophoresis. Analytical Chemistry, 65, 3454-3459. http://dx.doi.org/10.1021/ac00071a020

[8] Tiefenthaler, K. and Lukosz, W. (1984) Integrated Optical Switches and Gas Sensors. Optics Letters, 9, 137-139. http://dx.doi.org/10.1364/OL.9.000137

[9] Mossman, M.A. and Whitehead, L.A. (2005) Controlled Frustration of Total Internal Reflection by Electrophoresis of Pigment Particles. Applied Optics, 44, 1601-1609. http://dx.doi.org/10.1364/AO.44.001601 
[10] Yeh, P., Yariv, A. and Hong, C.S. (1977) Electromagnetic Propagation in Periodic Stratified Media. I. General Theory. Journal of the Optical Society of America, 67, 423-438. http://dx.doi.org/10.1364/JOSA.67.000423

[11] Yariv, A. and Yeh, P. (1977) Electromagnetic Propagation in Periodic Stratified Media. II. Birefringence, Phase Matching, and X-Ray Lasers. Journal of the Optical Society of America, 67, 438-448. http://dx.doi.org/10.1364/JOSA.67.000438

[12] Kokubun, Y., Baba, T., Sakaki, T. and Iga, K. (1986) Low-Loss Antiresonant Reflecting Optical Waveguide on Si Substrate in Visible-Wavelength Region. Electronics Letters, 22, 892-893. http://dx.doi.org/10.1049/el:19860608

[13] Zourob, M., Simonian, A., Wild, J., Mohr, S., Fan, X., Abdulhalime, I. and Goddarda, N.J. (2007) Optical Leaky Waveguide Biosensors for the Detection of Organophosphorus Pesticides. The Analyst, 132, 114-120. http://dx.doi.org/10.1039/b612871h

[14] Delonge, T. and Fouckhardt, H. (1995) Integrated Optical Detection Cell Based on Bragg-Reflecting Waveguides. Journal of Chromatography A, 716, 135-139. http://dx.doi.org/10.1016/0021-9673(95)00611-P

[15] Fouckhardt, H. and Delonge, T. (1996) Optical Detector Including Bragg Waveguide Structure. US Patent Nr. 5572328.

[16] Fouckhardt, H. and Delonge, T. (1997) An Optical Detector Device. European Patent Nr. EP 0679881 B1.

[17] Fouckhardt, H., Grosse, A., Grewe, M. and Kuhnke, M. (2001) Micro Flow Modules with Combined Fluid Flow Channel and Optical Detection Waveguide-Hyper Rayleigh Scattering as a Case Study. Fresenius Journal of Analytical Chemistry, 371, 218-227. http://dx.doi.org/10.1007/s002160100963

[18] Stadler, S., Braeuchle, C., Brandl, S. and Gompper, R. (1996) Experimental Determination of the First Hyperpolarizability of New Chiral and Achiral Octupolar Tertiary Amines by Hyper-Rayleigh Scattering. Chemistry of Materials, 8, 414-417. http://dx.doi.org/10.1021/cm9503487

[19] Stadler, S., Braeuchle, C., Brandl, S. and Gompper, R. (1996) Determination of the First Hyperpolarizabilities of Octupolar Molecular Ions Made from Symmetric Cyanine Dyes. Chemistry of Materials, 8, 676-678. http://dx.doi.org/10.1021/cm950453g

[20] Steingötter, I. and Fouckhardt, H. (2005) Deep Fused Silica Wet Etching Using an Au-Free and Stress Reduced Sputter Deposited Cr Hard Mask. Journal of Micromechanics and Microengeering, 15, 2130-2135. http://dx.doi.org/10.1088/0960-1317/15/11/019

[21] Kimmle, C., Wolff, S., Doering, C. and Fouckhardt, H. (2013) Dependence of Fused-Silica Etch Rate on the Etch Mask Opening Diameter. Microelectronic Engineering, 112, 10-13. http://dx.doi.org/10.1016/j.mee.2013.05.006

[22] Grosse, A., Grewe, M. and Fouckhardt, H. (2001) Deep Wet Etching of Fused Silica Glass for Hollow Capillary Optical Leaky Waveguides in Microfluidic Devices. Journal of Micromechanics and Microengeering, 11, 257-262. http://dx.doi.org/10.1088/0960-1317/11/3/315 